\title{
BIOÉTICA: CONTEXTO E INCIDENCIAS EN EL ÁREA DE LA SALUD DE LA UAM
}

Por: Comunidad Académica de Bioética

Universidad Autónoma de Manizales

Bellasmin Arenas Quintana: Fisiotorapeuta imvestigación Docencią Universitaria. Postgrado

José Hoover Vanegas García. Filásofo, Magister Epistemalogia

Juan N Quintero González. Odontologo. Patólogo

Juan E. Camacho Perdomo. Odontölogo. Salubrista

César Vásquez. Antropólogo.Candidato Doctorado

Juan Guillermo López. Odontólogo. Investigación Docencia Universiłaria. Poshgrado

Juan Pablo Correa M. Odontälogo. Investigación Docencia Universiłaria. Postgrado

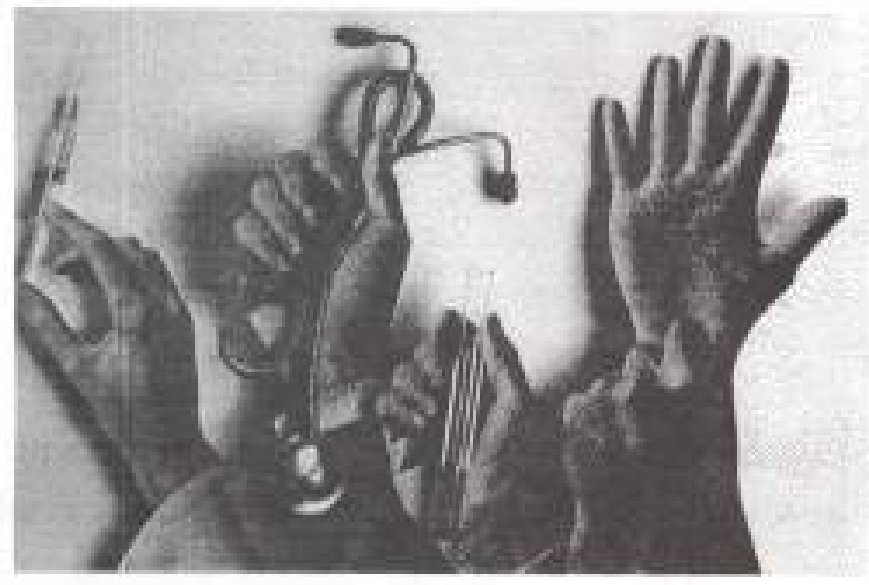

\section{NTRODUCCIÓN}

En la actualidat son mochos kos temas que se shordan desde el concepto do Bioctica; dentro de ellos tenemos los mas conscidos, coeno: la relación profestonal de salud, pacieste y aociedad. et trstamiesto del dolor indiferente al sufrimiento, la vida como detecho por encima de los valores y las relaciones humanas, la cutanasia, ef aborto, entre otros. Pero en las ultimas decadex, de accuendi a los muevos uvanoes 
cientifioas y tecinokigicos, se ha evidenciando otros problemas que ponen en tela de juicis la supreanacia de saber cientifico por encima de la vila y más precisamente por encima de la inter-personalidad, estos problemais son: la elonscion. las patentes genéticas, la manipulación transgénica, el transplante de órganos de acuerdo a necesidsdes patológicas, impiantaciones de silicenas con fines csticticos. La pregunta es: Latude el hombro inservenar bentro del desarmelo natural de ba vida sin perjudicar in humsnidad mismsa?.

Estos problemas es la sctualidad tienen muchs resonancią, pera la relación entre la salud y la etica no es un problema nuevo, podemos distinguir desde tiempos rematos indicios notmativos que eondicionan d tratamiento ite la vida y la integridad eorporal, por ejemplo: El eódigo de Hammurabi, elabonado 2500 afios antes de Criate dice; " Si un hownitor ha destruido el gio de wrnoble, su propia ojo Nenif destrida. Si ha rolo el hweso de wh noble, ni hueso send roto" Conno vemes en la cita, ls eual es solo ef comienzo de tal código. el intenta nomativizar por el castigo eorporal. e8 decir, es un condicionador que pane en juego la integridad fisiea.

De is misma forma encentramos el juramenio Hipocritica del säglo cuarto antes de Cristo, del cual citamos acilo el cuarto pairnfo: "No

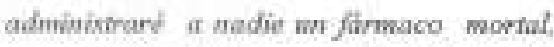

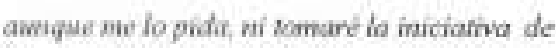
una sacgeroncla de eser zipor. Así miseno no

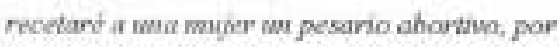
Al comarenk wivi y pracficant mi arie de fontia samia y pura." Vemos en este psesie del juramento hipocrifico, el cual todavia se actualiza como compromiso de los médicos nocifisos, la obligsturiedad de las profesionaks

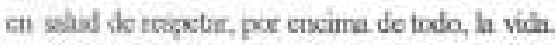

En este mismo sentido podrismos citar el juramento de Asaph, det siglo VI, o of jurametito de Maimonides del aiglo XII, de cualquier forma los peoblemas de le salud oon relacióa a la ćtica constituyen un hisloxial que cuenta desde que et hombre cayo en la cuentas de sa ser soeratico, desde que to humanidad se dio cucnta de que cra un cocrpo exptcosto a las inclemencias del mundo y de los trombres mismoc, la vida $y$ su perçuración sicmpre he sido un problema que al hambre ha querido resolver. y esto quiza, por la impotenia que semper ba fundado el feobmeno de la muerte en todos. los setes vives.

De acuerdo a este panorama de la relación entre lo normativo $y$ la vida queremos aventurarnos a una reflexión, en general, de la actualidad de ella, eon la eual prefendemos llamar la atención sobre su importancia parn el area de la galud en general, pero especialmente para in investigacion, $y$ esto de acuetulo a su aplicación en la Odontologia y la Fisioterapia Segün esto, buestra reflexión tendrá tres momendrs, primere: intentaremos acercarnos al concepto de Bicétien, tal conno se entiende en la actualidad, enfatizando en su earacteristica de cientifieidat, segundo nos aproximaremos a las reflexiones sabre las caracteristicas de la investigación en salod; y tercero: mostraranos algunos intertogantes de L, bioćtica y su pelación con la Odentologla y la Fisicterapia.

\section{APROXIMACIONES AL CONCEPTO DE BIOÉTICA}

En realidsd, los problemss que hemos mencionade en la introducxion a este trabajo son conocidos con el nomhre de ética médiea. La medicina oomes conocido por todos en el munda, es una disciplina pristana y de cicrta foema is madre de todas las aress de la salud. por ello es que la relación entere ćtica y salud estabs limitada a los descibrimientos médicos cono tal $y$ al cuidado de la vida humana de acusto a los cosocimientos begerobnicos de la época por la cual stravesabs la medicina.

Por otro Isdo la Bioética nace como un paraimetro o sensar para medir y regular los avances cientificrs, no sebla médiocos, sinn del àrea de la salud ex genesal y aun los probleinss. ambientales, es decir. In Bicetica, coeno su etimobogia misma lo advierse es una ciencia que pretende regular todas las actividades que pongan en peligro, no sóla la vida, sumo tambièn la calisdad de la misma.

La Bioćtica es una ciencia que intenta posicinnarse, no sále, en todos los àmbitns de la salud, como in medicina, la fisioterapia, la odontologis, sino que quiere ir mas lejos con la Ingenicria genética, no sólo en tanto aplicación a los seres hamabos, sino ets relación con todos low seres vivos, pero aûn màs, como consesuencia del arbitramiento de losavances cientifieos y la experimentación clíaica. La Bioctica debe axegurat d bietiestar secial de las commidades de todes kos seres vivos, en este sentido la Bioética es un cicricia futurista, ast lo deseribe Van Rensaelaer Potter en sa laber Bioethies. Bridge to the Future ex 1971 , este autor es quien introdtace a la cultura del conocimiento el têmino Biótres y st definieión reza de la siguiente forma: " $/ a$ ciencia del halaree entre el Hawhre y la Naturaleza, un puente hacha el fathero de la Inumarridind"

Otra definición que ha marcado el desarnollo de la Bioetion en las ûlimas décadas es la clahorada por el Instituto Kennedy de 
Fitos fundamentos o premisas conforman la base de sual quier estudio sohre el tema; adernas muestran la extensión que el mismo tienc, lo mismo que la importancia en el ámbito mumfial, onmo un grito de alerta frente a lafractura que esta podociendo el ser himano y, en general todo lo vivo, ai no nos oumprometemos con los problemas fundarientales de in Bioctica

Hay otro demento que no podernos dejar de meteionar, la Bsoćtica como todo estudio serio debe fence un métado, éste constituye d camino que se ha de recotrer para llegar a nuevos conocimientos, nuevos ptoductos que lo permitan a hr ciencia evoluciconar de acuerdo a la dinamies del mando tecnológice. Al respecto nomdice el profoser Spreccia: "Lametadologia

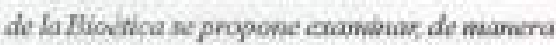
profund ty yachialeada la natumaleza del becho Aiomitileo (momento epistemologico): subriayar las implicaciames en el plano antropológico (mavaeno anitropológicol: e individuar las solucianes dicas y las festeficaciones de ardew racional que apeyan tales solucioner (macmento aplicativn) :-1

St aceptamos esta propuesta, el mittodo para Ia iaventigación en Bioética tiene tres momentos, el momento epistemológioo, el exal eonsiste en develar tos hechos bīonédicon, u partir de eatrueturas teóricas que permaitan analizar los criletios de verdad y de validor de los mármos, lo miamo que las estrueturas internas de las seorias sobre ef ferma en cuestión. El momento antropologico debe develarse eomo el eje del método Risoćtico, puesto que kvdos los problemas de esta cicneia parten del hombre, pero gravitando sobre el ser del mismo, tho solamente como estructurn biologich, munque también camo tal, nino, como sas que piensa, que sufte, en fín como un ser sintiente, oome hombre moral $Y$ por altimo el momento explicativo, este petmite justificu medtante la explicación racional y de aesuerdo a is experiencia cada uno de log problemes a los cuales se ve enfrentada la investigacián en esta área del saber ético

Tenemos cesatro argunnentos a nuestro modo de ver que le confieten seriedad a las investigaciones de Bioétics: In validez intemacional por las comunidndes cientifieas tanfo sociales como en cl ármbito de la salud, la delimitación del objeto de estudio, las bases o premises que posibilitan cuniquicr worcaniento a los problems de esta area de la etes y por ûltimo el método, el caial muestra el camino que se debe reoorrer. En este sentidin podemos afimar que la Bloctica no scolo se encarga de regular los avances cientifiens y tecnnlégiens, sino de vigilar reflexivamente fodo comportamiento que ponga en juego la vida en genenal, bo mismo que de set una ciencia futursta que esta velando por los intereses de las nuevas generaciones y pot último que tiene la calegoría de ciencia.

De scuerdo a exte panorama la Bioética es una ciencia que ha ganado un poesto dentro de la investigsción eientifien, y como tal debe ser uas reflexjón que contritiorya a la calidad de la vida en general, Instalemanos ahora en la investigaxiòn en salud y miremos alganes elementos que pueden servirnos pan tomar eonciencis de la labor que deben desempeilar, no sólo los cientifices de estu áren, sino todoe los seres humanos que tanto, activa coeno pasivamente son responsables del equilibrio entre los avances cientifiens y la calidad de vida.

\section{2. ÉTICA E INVESTIGACIÓN CLÍNICA}

L.a investigeción en las ôltimas décaites ha ganado mucbo terreao. la genética, tos transplantes, la robótica, la sintematización. El fin de fodios estos descubrimientas o adelantos cientificos, deberian contribuif al desarrollo humano, a la búsquedta de la califad de vida; sin eintorgo to que nos muestra la vids cobidiant es que estos nocvios hallaggos han cobrado valor por el aso del poder, piénsese en la bornba atcómica de Hirochirna y Nagrakici. los experimentos de la segunda guera muodial. b llamadacugenenes (d buen origen) en pembere del cual no fueron pocas las pernonas esterilizadas tanle en Norte America come en Alemania, en nombre de la perfecosión comonal

Futa en cuanto a los productos cientificos, pero dotris de ellos se esconde todo un arsenal de procesos, la investigación de: avevas tecnologias, en et ámbito de la salud exige involuchat, dentro de la misma seres vives, esto impliea que se ponga en rietgo la vids en general y a los seres bumanos en erpocial. Por esto ha sido necesario ereas nuevos parametros regulativos frente a la insaciable búsquedx de nuevos conocirricatos de los seros humanos.

Esto signifies que si bien los avanees cientifieos paeden contribuir al bienestar de Is humanidad, de la misma forms pueden contribair a la extinción tetal de la mismm; por esto se haes necesario las reflexiones eticas que este tipo de procesos debe genenut. 
Es asti como desde el código de Nurentberg, en la declaración de Helsinki (1), se permitiô fundamentar los parametros éticas indispenables en la realizaciòn de investigasiones eon sujetos, cuando el fin es kerapcutiso of et simplemente cientifioc.

Las valorest en las cualest se fundumenta esta decluración, rafican en considerar al hombre como persona y no como objeto, e igualmente ccnsiderar miempre el libre consentimiento de la persona sujeto de la experimecritación y la no fenunctas a los derechas deatro de su propia ccemanidad. Esta declaración no exonets a los medicos investigadores de fa responsabilitad en los ànbitos moral, social y legal de los procodimientes adelantados en cada institución y pais.

Postetiormente so implementaron unas guias de buena practios climica(2) que permitieron regular la idoneidad en los sspectos operatives de los protocolos de investigación, y kas aspoctns éticos pura el diseìn, eondusción, registro y reporte de entudios clinicos de uvestigaciōn con seres humanos. Estas guias fueron preparadas bajo el ausprcio del comité internacional de armonizaciós, sobre los requerimientos teenicos para el registro de fümacos do vas humano. Incluye representsntes de la Uniôn Europea, EEUU y Jap̧ón y adoptado igualinente por los paises asociados a la OMS

Las utiliación de aerer vivos en general y seres humanos en espocial en las investipaciones tanto en el f́mbito cjentifico como en el profesoonal, se convierte en una patadoja en donde so ponen en juepo doc aspectos: primero la evoluciòn tecno-cientifica, esto es, los avanoes en el concecimiento del funcionamiento hiologgioo de los setes vivos. Estos avmoes, como ef desculanmiento det genoma humano, no son en ấ mismos buenos, puesto que la ciencia no tiene cornotaciones morales, $y$ mosnos los debe tener los nuevos descubrimientos, ahon bien, comna se poode evolucionar en el conocimicnto de las estructurns. las causas y low efoctor de la vida, in no es estudiando la vida miama. Este parece ser un argumento necesario pasa hacer de lodo lo vivo un ovjeta de estudian.

El vegundo elemento de ia paradoja es jen que medids hacer de toda lo vivo $y_{i}$ esgocialirente de las persanas, un objeto de estudio, no es convertir lo vaviente en un útil, ento es, en un medio?. Hacer de ka seres humanos medios o instrumentos, significa, esclavizat el set nismo de la hamanidad a sus condiciones biobogicas, esto cs, jugat eos la dipnidad de las persomas. La paradoja consiste, en la necosidad
Ue la investigación, peto en la instruinentalización de los serea vivos. o en el estatiseno de la investignción en favor del respeto de lo viviente y en eipecial de les seres humanos.

Sis embargo, esta paradojil parece ser resuelta pot medio de lat normatividades que uns sociedad mcional inpotie a low cientifions y a todas ins persenss que, de una u otra forma, están involucrados con el áres de la silud. Estas reglas pormativas intentan mediar en la parndoja; desde ellas ae intenta no obstaculizar la investigación pero A la vez respetar los derechos y detares mismos de los sujetes actores en la bisqueda del conocimientor, esto justifica que en todo pais debe existir un reglamento ético que regule estall practicas, A estas condiciones se adbiere Colombin.

El ministeris de salud de Colombia mednante la resolución 8.430 de 1993, establece les nomas cientificas, administrativas y fécrieas para Is investigsción en salud, la eual tiene en cuenta los aiguientes criterios primero, contar con los principios éticos y cientificos que ta juatifique, esto ifenifica que toda investigación en aslud dobe regime por utas reglas que delimiten su experimentar mimo en la medida en que se pongs en juego, no wolo la integridad biologica de los seres vivos sino también la dignidad de las personas, contatar con los prificipeos cticos debe asezumer in seriodad moral de la investipación en salud.

Segando, que perenalezes la scguridad de los indivaluos participuegter pensar en la seguridad es prever la permaneneia de las personna, como seres integrales, en in ambiente adecusde, es decir, ascgurar el tituro en el presecte por medio del condicionamieno de los aceos que en algun sentida puedan dañar la dignided humana. Tercero, que tenga un consenteniento informado por escrito, esto quiere dseir, que ha voluntad de quien se expone a servir eomo elemento de inveafigacion pueda o no serib en el momenio que el quiera y dejar de serko cuando de mismo ks preficre, el conwentimicnto bene implicito la participación activa de las sujetos dentro de la investigación como bal, per clik deben ver informados de la imvestiosión y de los reseos que pueden oorrer.

Cuarto, que los investigadores setilen hejo la responsabelidad de una institución, sote requerimiento aseguna la legalidad de la mveatigación lo mismo que ls panuatia de quien se ecpore. Esta legislosont actualmente es inisuficiente, la que pernaite la posibilidad de interpretacion por parte de los diversos investigadoes, y adiciontalmente no establoce las kanesones es caso que se incumplan cicrtos parminctras. 
En la whatidad, de 230 isntituciones anesondas por el minásterio de salud para la confivemactón de comité de Bicetica, solo cinco funcionan de acuerdo eon la normatividad vigente. Como funcionses de dsches conté esta of evalust, discusar, aprobar, solieitar modifieación, negar aprobaciōn y geguir protcocolos de investigación en rujetos humunos para salvaguardar los derechos, la seguridad y el bieostar de los pacientes. La ocunformacḱn y funcionamiente de este comité so ve limitado pot aspectos de infraestruetion, recursos humanos $y$ eocancimicos que san de diffeil cumplimienta en peiquelas instituciones a de investipsdoes: indcperdientes. Fistos estain conformadas por einco expertos uno de ireas no cientificas, otro extrainstituciocual, de ambos sexos, y que ne tengan intereses en condicth.

El profesoe Gonealo Herranz de la Univeraidad de Naharra (Eapata) y miembro del comite ejecutivo de Hoodbca de la UNESCO, afima sobofe las funciones de los comités. ad onruts de sukfuncicrast fipicas de wherar de nesposuder a las consitilas que be le fincen rohre casas y

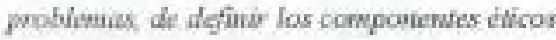
do has directrioes y profocolos de actwacion! Los romités contribuyes decisinamente a eriear.

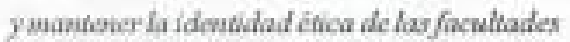
de modicina y de sux horpitales. Hoy, un hospital que nermencia a suser in comide do

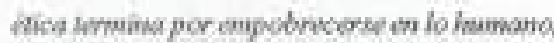

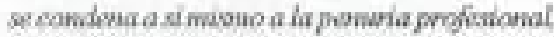
se arrieswa af perpetuar sus errones, paes carece de ese eficaz mocanismo para corregiriass"

Los comites deben seyulan, coms lo advierte ef proteser de Nabarra, las acesones tanto de imlervención como de investigación en el firea de la saluat, de squi la mportancra de los comilés al interior de las instituciones que de ura uoora forma tienen relación con la salud bumama.

\section{Aleunas IMPLICACIONES DE LA ODONTOLOGÍA Y LA FISIOTERAPIA DESDE LA BIOÉTICA}

De aeixedo a los argumentus expuestos an la primem parte de este trabogo la Bioctica posec kejitimialuf acadèmica cr cl ànhito internucional y validez cientifics de scuerdo a ha detimitación del objeto de estudio, sus fundamentos y la metodologis propia de la misma to mismo que la feglamentsción det ministerio de salud 8430 pan lairivestiqución en el area de la salud. estos elementos son suficiontes parn afirmar que la Bioébies es un tema que debe ser estudiado en ta UAM, poes sus elementios pueden facilitar, no sôlo el apoyo a la investignoión en las dreas de la salud, sino cstodar sus propios temas, tales como la explosión demografien en relación eon la calud y los derechos Humanos, los avances teconokigices en toda las eaferas y su incidencia en et desartolio humano, lia labor que dete desempeñar los profesionales en la vida cocidiana de acuerdo a la bisqueda de la calidad humana, entre otros.

En este apartado soblo intentaremos reflexionar, sobre algunos problemas que competen directamente en la Odontologia y la Fisioterapia, esto con el fin ide resslitar is inpertancia en la Universidsd de reflexionat sohre temas de is Bioćticu con relación a estas disciplinas.

Si bien la Bootica tiene incideroca en kodos los campos en donde so pone en juego, tanlo in vida comols dignidad humank, aqui sblo sumos a abordar la reflexión sobre las implicaciones en la Odontologia y la Firioterapia

La primen se suexnihe dentro de los limites de la salud estamatológica, la odantakogis debe velar por el bienestar oral tanto desde el ámbito Biolópico y sociocultural, como desdo la

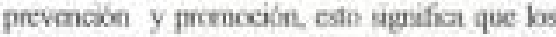
odontoblogos estain obligados por las nonnas morales a ser promotores en la comuadad de actinutes crales siladables. asi se ufirma ar be Principios Interisacionales de ética para la

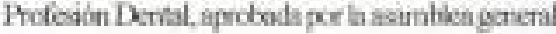
de h HDI en Seul, Ceran, septembre de 1997, la

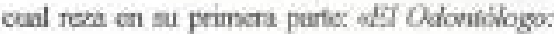

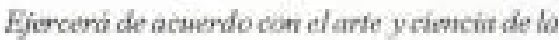
Odoniologia y principias humanisticos Protegená la sulued Bacodertal de los parientis:

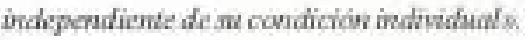

De neuerdo con esta aproximación 1 la Odostrologia, son muchos los problemas que podemos endenciar deide esta ires del siber de la salud, por qeanplo in relación entre el protesconal de la Odontodogia y los pacientes. ef iratamiento del sufrimiento, ef respeto, la tolerancia, el consenstimiento informalo, etc: cl uso de radiografias y has barreras de seguridad culkes son las condiciones böblogicas a las que se expooen los estudiantes en el uso de cotas técenicns, cuáles san los criterios de responsabilidad, eual es la responsabilidad frenie al iratamiento de la ortodoncia de us

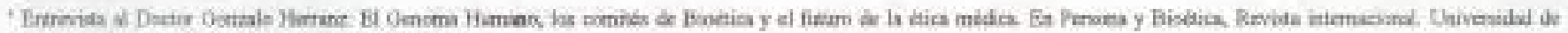

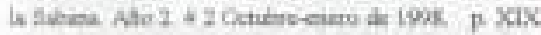


odontalogo expecialéta en esta disciplina y la de un Odontólogo general que elabore las mismas intervenciones; cual es la actitud de los profecionales de la sahud frente al virus VIH-Sida, cusies sue las relacioocs y las đfferencias entre in intervención odontologien de un profesional y un emporice, o los liamados Toguas":

De in minma Fonma podemos pensar la Firioternpia y sus unpleaciones Biocticas. Tornando. cn cuenta que la interexpción se asume como el ciercicio pofecuional en los deferentes campos de acxión, es dectr, en acciones orieatadas desde la promoción y proteceión. especifica tempétitica y rohabilitación, entro otras, tendiente a resolver tia probiematica de la salud desde lis perspectiva del movimiento corponil humsabo, spareeen muchos problemas que tienen incideckis decitro de la Bioetica de has cusies podernos enunciar la eugenesis, las poìtioas que exisfieton ent Nartesmetrca y. Alemana en la primeda mitad del siglo $\mathrm{XX}$; enterilizar a las personas pon disminución de la actividad para cyitar la descendencia de estas personat. jerra que un ser humano no tiene dercetho a procrear, por el sólo hecho de tener una malformación genétien? este es us probiems, que si bien no compele implicitamente a In intervencice fisiotempeuta, a tiene coinsecuencins en ella.

Otros probiemas que competen direetamente a la Fisioterapia son: In crecacia culturales que minimizan a las persona con dismiaución de la actividad, los valores negativos, como pesar, como impotencis, entre ofros, la igualdad de condiciones no solo para la actividad disria buo para las oportunidedes de cmpleo; de educeción ele.

El ejervicio de penetrar en estos problemas exige la metodologia, antes expuesta, el momento epistemológies, en donde se aclaren fon hecbos de una forna objesva y noutra, mirando adecruis sus estructuras teóncas y sus pngines. El uxamento astropologico, en donde hay que relacionar los hechos a investigar $y$ las roperousiones que ds tiene en la finrmación cultural del los hombres y de las mujeres lo mismo que la incidencia en of desartollo de nuevas entidades normativas que regulen el comportarmiento del hombre en general. $Y$ el momento explicativo, en donde hay yque relacionar las exkceptos fundamentales de la ética, tsles uner las norusas, las reglas, los vulores, los fines individuales y eolectivos, todo case a la lue det desarsollo harnan y la calidad de la inisma.

\section{BIBLIOGRAFIA}

HANNA, Thomas La Rebelion de los Cuerpos. Borcelonax Plazay Janes, S, A. 1972 .

Milani Marco y ntros. Elewertas para la ersethanza de la Bioetica. Caleceión Bios y ethos. Ediciones el hosque. Universidad ef bosque. Bogota 1998

Maturana Humberto y otros. Bioetica. La calidad de vida en el sigio XXT. Colección bios $\mathrm{y}$ ethos. Ediciones el Bosque. Universidad el Bosque. Bogotá 1995

Equobar Jaime Dimensianes Ontaibgicas del cuerpo. Coleosiobn bö y ethos. Ediciones el bosque. Universidad ef Bosque. Bogota 1998

Autares Varios. Codagos, counumbas y dectanaciones de èlica mèdicu, arfarmeria y bioctica. Colections bios y ethos. Ediciones el bosque, Universidad del bosque. Bogoti 1998

Estobar Jaime y otros. Probleness de dié aplienda. Coleccida bics y ethos. Ediciones el bosque. Universidad del bosque. Bogota 1997

HOTIJS Cilbert. EI Paradigina Biodtico. Haroekona: Antropos. 1991.

BASSO, M. Domingo. Nacer y Morir con Dignidad. Extudias de Buactica Confomponinea. Bogotá Colomba, Segunda Edieión. Paulinas. 1950 .

MAINETTL Jose Alborto. Biodica Ficta, La Plata. Argentina: Quirón. 1993 Antropohioetica. La Plata. Argentine: Quirin, 1995.

SEGRECCIA. Flio, Las Desafios de la Ricuitica Hoy. Santafe de Bogoth: Universidad de la Sabana, 1995

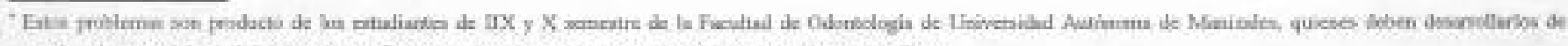

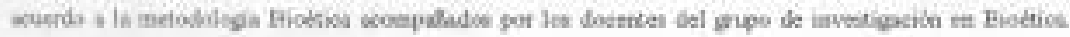

